KYUNGPOOK Math. J. 55(2015), 267-277

http://dx.doi.org/10.5666/KMJ.2015.55.2.267

pISSN 1225-6951 eISSN 0454-8124

(C) Kyungpook Mathematical Journal

\title{
Vertex Antimagic Total Labeling of Digraphs
}

\author{
J. PANDimadevi* \\ Department of Mathematics, EMG Yadava women's College, Madurai-625 014, \\ Tamilnadu, India \\ e-mail : pandimadevi87@yahoo.com \\ S. P. SubBiah \\ Department of Mathematics, Mannar Thirumalai Naicker College, Madurai-625 \\ 004, Tamilnadu, India \\ e-mail : jasminemtnc@gmail.com
}

Abstract. In this paper we investigate the properties of $(a, d)$-vertex antimagic total labeling of a digraph $D=(V, A)$. In this labeling, we assign to the vertices and arcs the consecutive integers from 1 to $|V|+|A|$ and calculate the sum of labels at each vertex, i.e., the vertex label added to the labels on its out arcs. These sums form an arithmetical progression with initial term $a$ and common difference $d$. We show the existence and nonexistence of $(a, d)$-vertex antimagic total labeling for several class of digraphs, and show how to construct labelings for generalized de Bruijn digraphs. We conclude this paper with an open problem suitable for further research.

\section{Introduction}

In this paper, we consider only finite directed graphs which admit loops but no multiple arcs. The vertex and arc sets of a digraph $D$ are denoted by $V(D)$ and $A(D)$ respectively and we let $|V(D)|=p$ and $|A(D)|=q$. For graph theoretic notations, we follow $[7,13]$. A labeling of a graph $G$ is a mapping that carries a set of graph elements, usually vertices and or edges into a set of numbers, usually integers. For a survey of magic and antimagic labelings one can refer to Gallian [9] and Baca et al. [2], In particular, antimagic labelings for certain classes of graphs were studied in $[3,4,5,10]$. In [12], Hartsfield and Ringel made a conjecture on vertex-antimagic labeling and Martin Baca proposed a conjecture about

\footnotetext{
* Corresponding Author.

Received March 3, 2014; revised September 3, 2014; accepted October 17, 2014.

2010 Mathematics Subject Classification: 05C78, 05C20.

Key words and phrases: $(a, d)$-vertex antimagic labeling, Generalized de Bruijn digraphs.
} 
edge-antimagic vertex labeling [1]. Thirusangu et al. [11] studied the super vertex $(a, d)$ - antimagic and vertex-magic total labelings for a particular class of Cayley digraphs.

The de Bruijn digraph defined in [8] has been noted as an interconnection network for massively parallel computers because of its good properties such as small diameter, high connectivity and easy routing (see [6]). The generalized de Bruijn digraph $G_{B}(n, d)$ is defined in $[6,8]$ by the congruence equations as follows:

$$
\begin{gathered}
V\left(G_{B}(n, d)\right)=\{0,1,2, \ldots, n-1\} \quad \text { and } \\
A\left(G_{B}(n, d)\right)=\{(x, y): y \equiv d x+i(\bmod n), 0 \leq i \leq d-1\},
\end{gathered}
$$

where $n$ and $d$ are positive integers with $d \leq n$. Though different kinds of labelings were studied by many author and many conjectures were made for different subclasses of graphs and digraphs, the labeling of some well-known digraphs, namely generalized de Bruijn digraphs, has not been investigated. Now, we introduce some defnitions which are needed to formulate this article.

Definition 1.1. The vertex-weight of a vertex $v$ in $D$ under a total labeling is defined as the sum of the label of $v$ and the arc labels corresponding to all the out-arcs of $v$. If all vertices in $D$ have the same weight $k$, we call the labeling vertex out-magic total labeling and we call $k$ a magic constant. If all vertices in $D$ have different weights, then the labeling is called vertex out-antimagic total labeling. A digraph $D$ which admits vertex out-antimagic total labeling is called vertex outantimagic digraph.

Definition 1.2. A bijection $f: V(D) \cup A(D)$ to the consecutive integers $1,2, \ldots, p+q$ is called an $(a, d)$ - vertex out-antimagic total labeling (VOATL) of $D$ if the set of vertex weights is $W=\{w t(x): x \in V(D)\}=\{a, a+d, \ldots, a+(p-1) d\}$ where $a$ and $d$ are integers and $w t(u)=f(u)+\sum_{(u, v) \in A(D)} f((u, v))$. A digraph $D$ which admits $(a, d)$-vertex out-antimagic total labeling is called $(a, d)$-vertex out-antimagic digraph.

Definition 1.3. An $(a, d)$-vertex out-antimagic total labeling is said to be a $V$ super $(a, d)$-vertex out-antimagic labeling if $f(V(D))=\{1,2,3, \ldots, p\}$. A digraph $D$ which admits $V$-super $(a, d)$-vertex out-antimagic labeling is called $V$-super $(a, d)$ vertex out-antimagic digraph.

Definition 1.4. An $(a, d)$-vertex out-antimagic total labeling is said to be a $E$-super $(a, d)$-vertex out-antimagic labeling if $f(A(D))=\{1,2,3, \ldots, q\}$. A digraph $D$ which admits $E$-super $(a, d)$-vertex out-antimagic labeling is called $E$-super $(a, d)$-vertex out-antimagic digraph.

Note that similar definitions hold for in-magic.

In our terminology, we call $(a, d)$-vertex out-antimagic total labeling as $(a, d)$ vertex antimagic labeling.

\section{Basic Counting}


In this section, we estabilish the bounds of $a$ and $d$ for any digraph $D$.

Let $\delta^{+}, \Delta^{+}$be the minimum and maximum out-degree of a digraph $D$ respectively. The minimum possible weight on a vertex is at least $1+2+\ldots+\left(\delta^{+}+1\right)$ and hence

$$
a \geq \frac{\left(\delta^{+}+1\right)\left(\delta^{+}+2\right)}{2}
$$

From the Theorem 3.7, we observe that the lower bound is tight. Similarly, if $\Delta^{+}$ is the largest degree, then the maximum vertex weight $l$ is not more than the sum of the $\Delta^{+}+1$ largest labels $p+q, p+q-1, p+q-2, \ldots, p+q-\Delta^{+}$. A simple calculation shows that

$$
l \leq \frac{\left(\Delta^{+}+1\right)\left(2(p+q)-\Delta^{+}\right)}{2} .
$$

Using $a$ and $l$, we have

$$
d \leq \frac{\left(\Delta^{+}+1\right)\left(2(p+q)-\Delta^{+}\right)-\delta^{+}\left(\delta^{+}+1\right)}{2(p-1)}
$$

In Section 3, we discuss $(a, d)$-vertex antimagic labeling in digraphs. Section 4 contains a discussion of $(a, d)$-vertex antimagic labeling in generalized de Bruijn digraphs. Section 5 contain the conclusion and an open problem.

\section{3. $(a, d)$-Vertex Antimagic Digraphs}

In this section, we find some classes of digraphs which admit $(a, d)$-vertex antimagic labeling and find some classes of digraphs which do not admit this labeling. Also, we establish the relation between magic and $(a, d)$-vertex antimagic labeling.

Proposition 3.1. If an $(a, d)$-vertex antimagic digraph $D$ is vertex magic, then $\frac{2 a+(p-1) d}{2}$ is an integer and the magic constant is $k=\frac{2 a+(p-1) d}{2}$.

Proof. Let $f$ be the $(a, d)$-vertex antimagic labeling defined on $D$ with $p$ vertices and $q$ arcs. The sum of all vertex weights is given by $a+a+d+a+2 d+\ldots+a+(p-1) d$. we have, $k=\frac{a+a+d+a+2 d+\ldots+a+(p-1) d}{p}$

Therefore, $k=\frac{2 a+(p-1) d}{2}$.

Theorem 3.2. Every directed path $\overrightarrow{P_{n}}$ is $(n, 2)$-vertex antimagic.

Proof. Let $\overrightarrow{P_{n}}$ be the directed path with vertex set $\left\{v_{1}, v_{2}, \ldots, v_{n}\right\}$ and arc set $A(D)=\left\{a_{i}=\left(v_{i}, v_{i+1}\right): 1 \leq i \leq n-1\right\}$. Let $f$ be a bijection from $V(D) \cup A(D)$ to the consecutive integers $\{1,2, \ldots 2 n-1\}$.

Label the vertices and edges as follows:

$$
\begin{aligned}
f\left(v_{i}\right) & =i, \forall i, 1 \leq i \leq n . \\
f\left(\left(v_{i}, v_{i+1}\right)\right) & =f\left(a_{i}\right)=n+i, \forall i, 1 \leq i \leq n-1 .
\end{aligned}
$$


Clearly $w t\left(v_{1}\right)=n+2, w t\left(v_{2}\right)=n+4, w t\left(v_{3}\right)=n+6, \ldots, w t\left(v_{n-1}\right)=3 n-2$, $w t\left(v_{n}\right)=n$.

Therefore, $f$ is an $(n, 2)$-vertex antimagic total labeling of $\overrightarrow{P_{n}}$.

Theorem 3.3. The directed cycle $\overrightarrow{C_{n}}$ is $(n+2,2)$-vertex antimagic for all $n$.

Proof. Let $\left\{v_{1}, v_{2}, v_{3}, \ldots, v_{n}\right\}$ be the vertex set of $\overrightarrow{C_{n}}$. Define a bijection $f$ from $V(D) \cup A(D)$ to the consecutive integers $\{1,2, \ldots 2 n\}$ as follows:

$$
\begin{gathered}
f\left(v_{i}\right)=i, \forall i, 1 \leq i \leq n . \\
f\left(\left(v_{i}, v_{i+1}\right)\right)=n+i, \forall i, 1 \leq i \leq n-1 . \\
f\left(\left(v_{n+1}, v_{1}\right)\right)=2 n
\end{gathered}
$$

The weights of the vertices $v_{1}, v_{2}, v_{3}, \ldots, v_{n}$ are $n+2, n+4, \ldots, 3 n$ respectively. Therefore, $f$ is an $(n+2,2)$-vertex antimagic total labeling of $\vec{C}_{n}$.

Note that double path $\overleftrightarrow{P_{2}}$ with 2 vertices is $(4,2)$-vertex antimagic.



Figure 1: Double path $\overleftrightarrow{P_{2}}$

Theorem 3.4. The Double paths $\overleftrightarrow{P_{n}}, n \geq 3$ is not $(a, d)$-vertex antimagic.

Proof. Let $D$ be the double path with vertex set $\left\{v_{1}, v_{2}, v_{3}, \ldots, v_{n}\right\}$. Clearly $|V(D)|=$ $n$ and $|A(D)|=2 n-2$. We assume that $D$ is $(a, d)$ - vertex antimagic.

Case (i). $n$ is odd.

Since D is $(a, d)$ - vertex antimagic, the weights of the vertices are $a, a+d$, $a+2 d, \ldots, a+(n-1) d$ and the sum of the weights is

$$
\begin{aligned}
S_{n}=\frac{n}{2}(2 a+(n-1) d) & =\frac{(3 n-2)(3 n-1)}{2} \\
2 a+(n-1) d & =\frac{(3 n-2)(3 n-1)}{n} \\
a & =\frac{(9-d)(n-1)}{2}+\frac{1}{n}
\end{aligned}
$$


Since $n$ is odd and $n \geq 3$, the first term is an integer but the second term is not an integer.

Case (ii). $n$ is even.

By similar arguement as in Case (i), we have

$$
a=\frac{(9-d)(n-1)}{2}+\frac{1}{n}
$$

Since $n \geq 4$ is even, the first term is an integer only when $d$ is odd but the second term is not an integer.

Theorem 3.5. If every vertex of a digraph $D$ has the same out-degree, then $D$ is $\left(\frac{p\left(\delta^{+}\right)^{2}+p \delta^{+}+2 \delta^{+}+2}{2}, \delta^{+}+1\right)$ - vertex antimagic.

Proof. Let $V(D)=\left\{v_{1}, v_{2}, v_{3}, \ldots, v_{p}\right\}$ be the vertex set of $D$. Define a bijection $f: V(D) \cup A(D)$ to $\{1,2, \ldots, p+q\}$ by $f\left(v_{i}\right)=i$, for all $v_{i} \in V(D)$. The arc set of $D$ can be written as $A(D)=\cup_{k=1}^{p} A_{k}$ where $A_{k}=\left\{\left(v_{k}, x\right): x \neq v_{k}, x \in V(D)\right\}$. The labeling for the $\operatorname{arcs}$ of $D$ is given in Table 1 .

Using Table 1, we obtain

$$
\begin{aligned}
a & =1+p+1+2 p+1+3 p+1+\ldots+p\left(\delta^{+}-1\right)+1+p \delta^{+}+1 \\
& =\left(\delta^{+}+1\right)+p\left(1+2+3+\ldots+\delta^{+}\right) \\
& =\frac{p\left(\delta^{+}\right)^{2}+p \delta^{+}+2 \delta^{+}+2}{2}
\end{aligned}
$$

and

$$
\begin{aligned}
l & =p+2 p+3 p+\ldots+p \delta^{+}+p\left(\delta^{+}+1\right) \\
& =\delta^{+}+1+\frac{p \delta^{+}\left(\delta^{+}+1\right)}{2} \\
& =\frac{p\left(\delta^{+}\right)^{2}+3 p \delta^{+}+2 p}{2}
\end{aligned}
$$

Clearly,

$$
\begin{aligned}
d & =\frac{l-a}{p-1} \\
& =\frac{\frac{p\left(\delta^{+}\right)^{2}+3 p \delta^{+}+2 p}{2}-\frac{p\left(\delta^{+}\right)^{2}+p \delta^{+}+2 \delta^{+}+2}{2}}{p-1} \\
& =\frac{2 p \delta^{+}+2 p-2 \delta^{+}-2}{2(p-1)} \\
& =\delta^{+}+1, \text { which is the desired property. }
\end{aligned}
$$


Table 1: Arc labels.

\begin{tabular}{|c|c|c|c|c|c|c|c|}
\hline$A_{k}$ & \multicolumn{7}{|c|}{ Arc labels for $\operatorname{arcs}$ in $A_{k}$} \\
\hline$A_{1}$ & $p+1$ & $2 p+1$ & $3 p+1$ & $4 p+1$ & $\ldots$ & $p\left(\delta^{+}-1\right)+1$ & $p \delta^{+}+1$ \\
\hline$A_{2}$ & $p+2$ & $2 p+2$ & $3 p+2$ & $4 p+2$ & $\ldots$ & $p\left(\delta^{+}-1\right)+2$ & $p \delta^{+}+2$ \\
\hline$A_{3}$ & $p+3$ & $2 p+3$ & $3 p+3$ & $4 p+3$ & $\ldots$ & $p\left(\delta^{+}-1\right)+3$ & $p \delta^{+}+3$ \\
\hline$\ldots$ & $\ldots$ & $\ldots$ & $\ldots$ & $\ldots$ & $\ldots$ & $\ldots$ & $\ldots$ \\
\hline$\ldots$ & $\ldots$ & $\ldots$ & $\ldots$ & $\ldots$ & $\ldots$ & $\ldots$ & $\ldots$ \\
\hline$A_{p-1}$ & $2 p-1$ & $3 p-1$ & $4 p-1$ & $5 p-1$ & $\ldots$ & $p \delta^{+}-1$ & $p\left(\delta^{+}+1\right)-1$ \\
\hline$A_{p}$ & $2 p$ & $3 p$ & $4 p$ & $5 p$ & $\ldots$ & $p \delta^{+}$ & $p\left(\delta^{+}+1\right)$ \\
\hline
\end{tabular}

Corollary 3.6. If every vertex of a digraph $D$ has the same out-degree and $\frac{\left(p \delta^{+}+p+1\right)\left(\delta^{+}+1\right)}{2}$ is an integer, then $D$ is vertex magic with magic constant $k=$ $\frac{\left(p \delta^{+}+p+1\right)\left(\delta^{+}+1\right)}{2}$.

Proof. By Proposition 3.1. and Theorem 3.5,

$$
\begin{aligned}
k & =\frac{a+l}{2}=\frac{2 p\left(\delta^{+}\right)^{2}+4 p \delta^{+}+2 p+2 \delta^{+}+2}{4} \\
& =\frac{\left(\delta^{+}+1\right)\left(p \delta^{+}+p+1\right)}{2}
\end{aligned}
$$

The lower bound for ' $a$ ' mentioned in Section 2 can be calculated as $\frac{\left(\delta^{+}\right)^{2}+3 \delta^{+}+2}{2}$. The following theorem describes the $(a, d)$-vertex antimagicness of a digraph when $a=\frac{\left(\delta^{+}\right)^{2}+3 \delta^{+}+2}{2}$.

Theorem 3.7. If every vertex of a digraph $D$ has the same out-degree, then $D$ is $\left(\frac{\left(\delta^{+}\right)^{2}+3 \delta^{+}+2}{2}, \frac{p\left(\delta^{+}\right)^{2}+2 p \delta^{+}+p-\left(\delta^{+}\right)^{2}-2 \delta^{+}-1}{p-1}\right)$-vertex antimagic.

Proof. Let $V(D)=\left\{v_{1}, v_{2}, v_{3}, \ldots, v_{p}\right\}$ be the vertex set of $D$. Define a bijection $f: V(D) \cup A(D)$ to $\{1,2, \ldots, p+q\}$ by $f\left(v_{i}\right)=(i-1) \delta^{+}+i$, for all $v_{i} \in V(D)$. The arc set of $D$ can be written as $A(D)=\cup_{k=1}^{p} A_{k}$ where $A_{k}=\left\{\left(v_{k}, x\right): x \neq v_{k}, x \in\right.$ $V(D)\}$. The labeling for the $\operatorname{arcs}$ of $D$ is given in Table 2 .

From Table 2, we see that

$$
\begin{aligned}
a & =1+2+3+\ldots+\left(\delta^{+}+1\right) \\
& =\frac{\left(\delta^{+}\right)^{2}+3 \delta^{+}+2}{2} \text { and } \\
l & =(p-1) \delta^{+}+p+(p-1) \delta^{+}+p+1+\ldots+(p-1) \delta^{+}+p+\delta^{+} \\
& =(p-1) \delta^{+}\left(\delta^{+}+1\right)+p\left(\delta^{+}+1\right)+\frac{\delta^{+}\left(\delta^{+}+1\right)}{2} \\
& =\frac{2 p\left(\delta^{+}\right)^{2}+4 p \delta^{+}+2 p-\left(\delta^{+}\right)^{2}-\delta^{+}}{2} .
\end{aligned}
$$


By using the value of $a$ and $l$, we have

$$
d=\frac{l-a}{p-1}=\frac{p\left(\delta^{+}\right)^{2}+2 p \delta^{+}+p-\left(\delta^{+}\right)^{2}-2 \delta^{+}-1}{p-1} .
$$

Table 2: Arc labels.

\begin{tabular}{|c|c|c|c|c|}
\hline$A_{k}$ & \multicolumn{4}{|c|}{ Arc labels for arcs in $A_{k}$} \\
\hline$A_{1}$ & 1 & $\ldots$ & $\delta^{+}$ & $\delta^{+}+1$ \\
\hline$A_{2}$ & $\delta^{+}+2$ & $\ldots$ & $2 \delta^{+}+1$ & $2 \delta^{+}+2$ \\
\hline$A_{3}$ & $2 \delta^{+}+3$ & $\ldots$ & $3 \delta^{+}+2$ & $3 \delta^{+}+3$ \\
\hline$\ldots$ & $\ldots$ & $\ldots$ & $\ldots$ & $\ldots$ \\
\hline$\ldots$ & $\ldots$ & $\ldots$ & $\ldots$ & $\ldots$ \\
\hline$A_{p-1}$ & $(p-2) \delta^{+}+p-1$ & $\ldots$ & $(p-1) \delta^{+}+p-1$ & $(p-1) \delta^{+}+p-1$ \\
\hline$A_{p}$ & $(p-1) \delta^{+}+p$ & $\ldots$ & $p \delta^{+}+p-1$ & $p\left(\delta^{+}+1\right)$ \\
\hline
\end{tabular}

Theorem 3.8. If every vertex of a digraph $D$ has the same out-degree $\delta^{+}$and $\delta^{+}$ is even, then $D$ is $\left(\frac{p\left(\delta^{+}\right)^{2}+2 p \delta^{+}+\delta^{+}+2}{2}, 1\right)$-vertex antimagic.

Proof. Let $V(D)=\left\{v_{1}, v_{2}, v_{3}, \ldots, v_{p}\right\}$ be the vertex set of $D$. Define a bijection $f: V(D) \cup A(D)$ to $\{1,2, \ldots, p+q\}$ by $f\left(v_{i}\right)=i$, for all $v \in V(D)$. The arc set of $D$ can be written as $A(D)=\cup_{k=1}^{p} A_{k}$ where $A_{k}=\left\{\left(v_{k}, x\right): x \neq v_{k}, x \in V(D)\right\}$. The labeling for the arcs of $D$ is given in Table 3 .

Using Table 3 , we find $a$ and $l$ as follows:

$$
\begin{aligned}
& a=1+2 p+2 p+1 \ldots+p \delta^{+}+p \delta^{+}+1 \\
&= \frac{p\left(\delta^{+}\right)^{2}+2 p \delta^{+}}{2}+\frac{\delta^{+}+2}{2} \\
&= \frac{p\left(\delta^{+}\right)^{2}+2 p \delta^{+}+\delta^{+}+2}{2} \\
& l=p+p+1+3 p+3 p+1+\ldots+p\left(\delta^{+}-1\right)+1+p\left(\delta^{+}+1\right) \\
&=2 p\left(1+3+5+\ldots+\left(\delta^{+}-1\right)\right)+p \delta^{+}+p+\frac{\delta^{+}}{2} \\
&=\frac{p\left(\delta^{+}\right)^{2}+\delta^{+}+2 p \delta^{+}+2 p}{2} .
\end{aligned}
$$

By using the value of $a$ and $l$, we have

$$
\begin{aligned}
d & =\frac{l-a}{p-1}=\frac{\frac{p\left(\delta^{+}\right)^{2}+\delta^{+}+2 p \delta^{+}+2 p}{2}-\frac{p\left(\delta^{+}\right)^{2}+2 p \delta^{+}+\delta^{+}+2}{2}}{p-1} \\
& =\frac{2 p-2}{2 p-2}=1 .
\end{aligned}
$$


Table 3: Arc labels.

\begin{tabular}{|c|c|c|c|c|c|}
\hline$A_{k}$ & \multicolumn{5}{|c|}{ Arc labels for arcs in $A_{k}$} \\
\hline$A_{1}$ & $2 p$ & $2 p+1$ & $\ldots$ & $p \delta^{+}$ & $p \delta^{+}+1$ \\
\hline$A_{2}$ & $2 p-1$ & $2 p+2$ & $\ldots$ & $p \delta^{+}-1$ & $p \delta^{+}+2$ \\
\hline$A_{3}$ & $2 p-2$ & $2 p+3$ & $\ldots$ & $p \delta^{+}-2$ & $p \delta^{+}+3$ \\
\hline$\ldots$ & $\ldots$ & $\ldots$ & $\ldots$ & $\ldots$ & $\ldots$ \\
\hline$\ldots$ & $\ldots$ & $\ldots$ & $\ldots$ & $\ldots$ & $\ldots$ \\
\hline$A_{p-1}$ & $p+2$ & $3 p-1$ & $\ldots$ & $p\left(\delta^{+}-1\right)+2$ & $p\left(\delta^{+}+1\right)-1$ \\
\hline$A_{p}$ & $p+1$ & $3 p$ & $\ldots$ & $p\left(\delta^{+}-1\right)+1$ & $p\left(\delta^{+}+1\right)$ \\
\hline
\end{tabular}

Theorem 3.9. If the following conditions are hold, then the digraph $D$ is $\left(\frac{p\left(\delta^{+}\right)^{2}+2 p \delta^{+}+\delta^{+}+2}{2}, 1\right)$-vertex antimagic.

1. Every vertex of $D$ has the same out-degree $\delta^{+}$

2. $p$ is odd

3. $\delta^{+}$is odd

Proof. Let $V(D)=\left\{v_{1}, v_{2}, v_{3}, \ldots, v_{p}\right\}$ be the vertex set of $D$. Define a bijection $f: V(D) \cup A(D)$ to $\{1,2, \ldots, p+q\}$ by

$$
f\left(v_{i}\right)= \begin{cases}\frac{i+1}{2}, & i \text { is odd } \\ \frac{n+i+1}{2}, & i \text { is even. }\end{cases}
$$

for all $v_{i} \in V(D)$. The arc set of $D$ can be written as $A(D)=\cup_{k=1}^{p} A_{k}$ where $A_{k}=\left\{\left(v_{k}, x\right): x \neq v_{k}, x \in V(D)\right\}$. The labeling for the arcs of $D$ is given in Table 4 .

Using Table 4,

$$
\begin{aligned}
a & =\frac{p+1}{2}+p+1+3 p+3 p+1+\ldots+p\left(\delta^{+}\right)+p\left(\delta^{+}\right)+1 \\
& =\frac{\delta^{+}-p+2}{2}+\frac{p\left(2\left(\delta^{+}\right)^{2}+2 \delta^{+}-\left(\delta^{+}\right)^{2}+1\right)}{2} \\
& =\frac{p\left(\delta^{+}\right)^{2}+2 p \delta^{+}+\delta^{+}+2}{2}
\end{aligned}
$$

By a similar argument as in Therorem 2.8., we get

$$
l=\frac{p\left(\delta^{+}\right)^{2}+\delta^{+}+2 p \delta^{+}+2 p}{2}
$$

and by using the value of $a$ and $l$, we have

$$
d=\frac{l-a}{p-1}=\frac{\frac{p\left(\delta^{+}\right)^{2}+\delta^{+}+2 p \delta^{+}+2 p}{2}-\frac{p\left(\delta^{+}\right)^{2}+2 p \delta^{+}+\delta^{+}+2}{2}}{p-1}=\frac{2 p-2}{2 p-2}=1
$$


Table 4: Arc labels.

\begin{tabular}{|c|c|c|c|c|c|c|}
\hline$A_{k}$ & \multicolumn{6}{|c|}{ Arc labels for arcs in $A_{k}$} \\
\hline$A_{1}$ & $2 p$ & $2 p+1$ & $4 p$ & $4 p+1$ & $\ldots$ & $p\left(\delta^{+}+1\right)$ \\
\hline$A_{2}$ & $2 p-1$ & $2 p+2$ & $4 p-1$ & $4 p+2$ & $\ldots$ & $p\left(\delta^{+}+1\right)-1$ \\
\hline$A_{3}$ & $2 p-2$ & $2 p+3$ & $4 p-2$ & $4 p+3$ & $\ldots$ & $p\left(\delta^{+}+1\right)-2$ \\
\hline$\ldots$ & $\ldots$ & $\ldots$ & $\ldots$ & $\ldots$ & $\ldots$ & $\ldots$ \\
\hline$\ldots$ & $\ldots$ & $\ldots$ & $\ldots$ & $\ldots$ & $\ldots$ & $\ldots$ \\
\hline$A_{p-1}$ & $p+2$ & $3 p-1$ & $3 p+2$ & $5 p-1$ & $\ldots$ & $p \delta^{+}+2$ \\
\hline$A_{p}$ & $p+1$ & $3 p$ & $3 p+1$ & $5 p$ & $\ldots$ & $p \delta^{+}+1$ \\
\hline
\end{tabular}

\section{The Generalized De Bruijn Digraphs}

Theorem 4.1. The generalized de Bruijn digraph $G_{B}(n, d)$ is $\left(\frac{p d^{2}+p d+2 d+2}{2}, d+1\right)$ - vertex antimagic.

Proof. From the definition of $G_{B}(n, d)$, we have $\delta^{+}=\left|N^{+}(v)\right|=d$ for all $v \in$ $V\left(G_{B}(n, d)\right)$. By Theorem 3.5, the result follows.

Theorem 4.2. The generalized de Bruijn digraph $G_{B}(n, d)$ is $\left(\frac{d^{2}+3 d+2}{2}, \frac{p d^{2}+2 p d+p-d^{2}-2 d-1}{p-1}\right)$-vertex antimagic.

Proof. From the definition of $G_{B}(n, d)$, we have $\delta^{+}=\left|N^{+}(v)\right|=d$ for all $v \in$ $V\left(G_{B}(n, d)\right)$. By Theorem 3.7, the result follows.

It seems to be difficult to find $\left(\frac{p d^{2}+2 p d+d+2}{2}, 1\right)$ - vertex antimagic generalized de Bruijn digraph $G_{B}(n, d)$. The total labeling assign integers $1,2,3, \ldots, p+q=n+n d$ to the vertices and arcs. So, we divide them into three categories.

1. $d$ is even.

2. $n$ is odd and $d$ is odd.

3. $n$ is even and $d$ is odd.

Theorem 4.3. The digraph $G_{B}(n, d)$ is $\left(\frac{p d^{2}+2 p d+d+2}{2}, 1\right)$ - vertex antimagic, if $d$ is even.

Proof. From the definition of $G_{B}(n, d)$, we have $\delta^{+}=\left|N^{+}(v)\right|=d$ for all $v \in$ $V\left(G_{B}(n, d)\right)$. By Theorem 3.8, the result follows.

Theorem 4.4. The digraph $G_{B}(n, d)$ is $\left(\frac{p d^{2}+2 p d+d+2}{2}, 1\right)$ - vertex antimagic, if both $n$ and $d$ are odd.

Proof. From the definition of $G_{B}(n, d)$, we have $\delta^{+}=\left|N^{+}(v)\right|=d$ for all $v \in$ $V\left(G_{B}(n, d)\right)$. By Theorem 3.9, the result follows.

Theorem 4.5. The digraph $G_{B}(n, d)$ is not $(a, 1)$ - vertex antimagic, if both $n$ and $d$ are even. 
Proof. Let $\left|V\left(G_{B}(n, d)\right)\right|=n$ and $\left|A\left(G_{B}(n, d)\right)\right|=n d$. We assume that $G_{B}(n, d)$ is $(a, 1)$-vertex antimagic. The weights of the vertices are given by $a, a+d$, . . , $a+(n-1) d$, the sum of the weights is given by

$$
S_{n}=\frac{n(2 a+(n-1) d)}{2}=\frac{(n d+d)(n d+d+1)}{2}
$$

After a simple calculation, we get

$$
a=\frac{3 d^{2}+2 d-n d}{2}+\frac{d(d+1)}{2 n}
$$

and is not an integer, since $d \leq n$.

\section{Conclusion and Scope}

The $(a, d)$-vertex anti in-magicness of digraphs can be discussed in a similar way. For a generalized de Bruijn digraph $(a, d)$-vertex anti in-magic labeling is same as $(a, d)$-vertex anti out-magic labeling and $(a, d)$-vertex anti in-magic labeling and $(a, d)$-vertex anti out-magic labeling is same for all regular digraphs.

In this paper, first we have introduced the concept of $(a, d)$-vertex antimagic labeling in digraphs and found some results on digraphs. Secondly, we introduced $(a, d)$-vertex antimagic labeling in generalized de bruijn digraphs.

Based on the Theorem 3.5, Theorem 3.7, Theorem 3.8 and Theorem 3.9, we have the following open problem.

Open Problem 1. For given integers $a$ and $d$, find all $(a, d)$-vertex antimagic digraphs.

Acknowledgments. The authors are thankful to the anonymous referee for his/her valuable suggestions to improve the quality and the readability of the article.

\section{References}

[1] M. Baca, Y. Lin, M. Miller and R. Simanjuntak, New constructions of magic and antimagic graph labelings, Utilitas Mathematica, 60(2001) 229-239.

[2] M. Baca, F. Bertault, J. MacDougall, M. Miller, R. Simanjuntak and Slamin, Vertex-Antimagic total labelings of graphs, Discussiones Mathematicae Graph Theory, 23(2003), 67-3.

[3] J. Baskar Babujee, Antimagic labeling on certain classes of graphs, Acta Ciencia Indica, Mathematics XXXI, (4)(2005), 1027-1030.

[4] J. Baskar Babujee, On a conjecture of Martin Baca about (a, 1)-edge-antimagic vertex labeling, in: 11th International Conference on Multidisciplinary Mathematics SCRA 2004, South Alabama University, USA and IET-Lucknow, India, 2004. 
[5] J. Baskar Babujee and N. Prabhakara Rao, Labelling in digraph, Journal of the Mathematics Education, 39(2)(2005), 73-8.

[6] J. C. Bermond, C. Peyrat, de Bruijn and Kautz, Networks: A competitor for the hypercube?, in: F. Andre, J. P. Verjus (Eds.), Hypercube and Distributed Computers, North Holland, Amsterdam, (1989), 279-293.

[7] G. Chartrand and L. Lesniak, Graphs and Digraphs, Chapman and Hall, BocaRoton,London, Newyork, Washington, D.C., (1996), 3rd Edition.

[8] N. G. de Bruijn, A combinatorial problem, Koninklijke Nederlandse Akademievan Wetenschappen Proc., A49(1946), 745-764.

[9] J. A. Gallian, A dynamic survey of graph labeling, Electron. J. Combin., 19(2012), \#DS6.

[10] K. Thirusangu, A. K. Nagar and R. Rajeswari, Labelings in Cayley digraphs, European J. Combin., 32(1)(2011), 133139.

[11] K. Thirusangu, J. Baskar Babujee and R. Rajeswari, On antimagic labelings in Cayley digraphs, International Journal of Mathematics and Applications, 2(13)(2009), 11-16.

[12] N. Hartsfield and G. Ringel, Pearls in Graph Theory, Academic Press, San Diego, 1990.

[13] A. M. Marr and W. D. Wallis, Magic graphs, Birkhauser, Boston, Basel, Berlin, 2013 (2nd edition). 